\title{
Effectiveness of EMDR in patients with substance use disorder and comorbid PTSD: study protocol for a randomized controlled trial
}

Ingo Schäfer ${ }^{1,2}$, Laycen Chuey-Ferrer ${ }^{3}$, Arne Hofmann ${ }^{4}$, Peter Lieberman ${ }^{4}$, Günter Mainusch ${ }^{3}$ and Annett Lotzin ${ }^{1,2^{*}}$ (D)

\begin{abstract}
Background: Eye Movement Desensitization and Reprocessing (EMDR) is an evidence-based treatment for PTSD. However, it is unclear whether EMDR shows the same effectiveness in patients with substance use disorders (SUD) and comorbid PTSD. In this trial, we examine the effectiveness of EMDR in reducing PTSD symptoms in patients with SUD and PTSD.

Methods/Design: We conduct a single-blinded RCT among 158 patients with SUD and comorbid PTSD admitted to a German addiction rehabilitation center specialized for the treatment of patients with SUD and comorbid PTSD. Patients are randomized to receive either EMDR, added to SUD rehabilitation and non-trauma-focused PTSD treatment (TAU), or TAU alone. The primary outcome is change from baseline in PTSD symptom severity as measured by the ClinicianAdministered PTSD Scale at 6-month follow-up. Secondary outcomes are change from baseline in substance use, addiction-related problems, depressive symptoms, dissociative symptoms, emotion dysregulation and quality of life. Assessments are carried out by blinded raters at admission, at end of treatment, and at 3- and 6-month follow-up. We expect that EMDR plus TAU will be more effective in reducing PTSD symptoms than TAU alone. Mixed models will be conducted using an intention-to-treat and per-protocol approach.
\end{abstract}

Discussion: This study aims to expand the knowledge about the effectiveness of EMDR in patients with SUD and comorbid PTSD. The expected finding of the superiority of EMDR in reducing PTSD symptoms compared to non-trauma-focused PTSD treatment may enhance the use of trauma-focused treatment approaches for patients with SUD and co-morbid PTSD.

Trial registration: German Clinical Trials Register: DRKS00009007; U1111-1172-9213. Retrospectively registered 01 Juni 2016.

Keywords: Posttraumatic stress disorder, Alcohol, Substance abuse, Addiction, Comorbidity, Eye movement desensitization and reprocessing, Trauma-focused treatment, Exposure-based treatment, Psychotherapy outcome research

\section{Background}

Up to $45 \%$ of the patients with SUD experience comorbid PTSD [1-3], indicating a clear need for PTSD treatment in this population. Although the importance of comorbid PTSD in the treatment of patients with SUD has been recognized [4], most SUD inpatient rehabilitation

\footnotetext{
* Correspondence: a.lotzin@uke.de

'Department of Psychiatry and Psychotherapy, University Medical Center Hamburg-Eppendorf, Hamburg, Germany

${ }^{2}$ Center for Interdisciplinary Addiction Research, Department of Psychiatry and Psychotherapy, University Medical Center Hamburg-Eppendorf, University of Hamburg, Martinistr. 52, Hamburg D-20246, Germany Full list of author information is available at the end of the article
}

centers in Germany do not offer integrated treatment for SUD and PTSD. When an integrated treatment is offered, non-trauma-focused interventions are predominantly used to address PTSD [5]. This current clinical practice in routine SUD healthcare is in contrast to the national and international guidelines [6-8] that recommend trauma-focused treatment for PTSD, which clearly yields higher effect sizes compared with non-traumafocused approaches. However, the evidence base of these guidelines is built on trials in which patients with PTSD and comorbid SUD have been almost continuously excluded $[9,10]$. 
First evidence exists that trauma-focused treatments, such as prolonged exposure [11], trauma-focused imaginal exposure [12] and structured writing therapy [13], may also be effective in patients with PTSD and comorbid SUD in reducing PTSD symptoms, although the evidence is not consistent [13-15]. This trials also found that trauma-focused interventions for patients with SUD and comorbid PTSD could be applied securely without compromising substance use outcomes $[14,15]$. This is an important result, because clinicians may hesitate to use trauma-focused therapy for patients with SUD and comorbid PTSD, because they might believe that eliciting intense emotions related to the traumatic event during trauma-focused treatment may increase the risk for relapse [16, 17]. EMDR is another trauma-focused treatment that has been shown to be effective in patients with PTSD alone $[6,18]$. So far, no RCT has been conducted that examined whether EMDR is effective in reducing PTSD symptoms in patients with SUD and comorbid PTSD.

Trauma-focused PTSD treatment among patients with SUD may also effectively reduce SUD symptoms $[19,20]$. Patients with PTSD and SUD report higher levels of craving in response to trauma-related cues [21] than patients with PTSD. Therefore, these patients might be more likely to use substances to regulate negative affective states associated with PTSD [20, 22, 23]. If negative affective states related to PTSD could be effectively reduced by trauma-focused treatment, SUD symptoms might also be reduced.

\section{Research aims and hypothesis}

The primary aim of this study is to investigate the effectiveness of EMDR, added to SUD rehabilitation and nontrauma-focused PTSD treatment (TAU), in reducing PTSD symptom severity at 6-month follow-up compared with TAU alone in patients with SUD and comorbid PTSD. We hypothesize that EMDR combined with TAU will lead to a significantly greater reduction of PTSD symptoms than TAU alone, when comparing PTSD symptoms from baseline to 6-month follow-up. As secondary outcomes, we examine the effectiveness of EMDR on substance use-related outcomes, depressive symptoms, dissociative symptoms, emotion dysregulation and quality of life. If this study shows that EMDR can be used effectively in patients with SUD and comorbid PTSD, the results of this RCT might encourage a more frequent use of evidence-based trauma-focused treatments for patients with SUD and comorbid PTSD.

\section{Methods}

\section{Design and study setting}

This study is a rater-blinded 2-arms RCT. Assessments are scheduled pre-treatment (T0), post-treatment (T1), at 3-month (T2) and at 6-month (T3) follow-up. Participants are randomly assigned to either the EMDR plus TAU group or the TAU group. Data is assessed at an inpatient rehabilitation center in Germany (AHG Clinic Dormagen). This center is specialized for the treatment of patients with SUD (primarily alcohol use disorders) and comorbid PTSD. The study is coordinated by a research team of the Center for Interdisciplinary Addiction Research (CIAR), University Medical Center Hamburg-Eppendorf, Germany. The coordinating site is responsible for the study design, the training of the personnel involved in the data collection, the preparation and supply of all study documents, study monitoring, study supervision, data management and analysis, and reporting of the study results.

\section{Participants}

We plan to recruit 158 adult patients with SUD and comorbid PTSD attending inpatient rehabilitation treatment from September 2015 to December 2017. Inclusion criteria are (1) age between 18 and 65 years; (2) DSM-5 diagnosis of a substance use disorder [24]; (3) DSM-5 diagnosis of PTSD or subsyndromal PTSD (criteria A and B and at least one of the criteria $\mathrm{C}$ to $\mathrm{E}$ ) [18]; (4) capable to comprehend and speak German; (5) informed consent to participate in the study. Exclusion criteria are (1) severe dissociative symptoms according to the Dissociative Experience Scale [25] (total score > 40); (2) acute suicidality; (3) acute psychotic symptoms; and (4) severe cognitive impairments.

\section{Interventions}

All patients included in this study participate in the usual treatment (TAU) of the SUD rehabilitation center provided for patients with SUD and comorbid PTSD. TAU includes SUD rehabilitation and non-traumafocused PTSD treatment. TAU is present-focused and includes the provision of knowledge, techniques and skills to better cope with PTSD symptoms and to prevent SUD relapse (psychoeducation about PTSD and SUD; resource activation, e.g. establishing positive activities; imaginative exercises [26]; acceptance- and mindfulness-oriented skills [27]; elements of Seeking Safety [28], non-trauma-focused CBT). A treatment protocol was created prior to the start of the study that defines the intervention elements of the non-traumafocused treatment. No processing of traumatic memories takes place in the TAU condition.

All study participants receive two 90-min and two 60min non-trauma-focused group therapy sessions per week. In addition to the non-trauma-focused group therapy, participants randomized to the TAU group additionally receive one 50 -min individual non-traumafocused therapy session per week; participants randomized 
to the EMDR group receive one 50-min individual EMDR session per week. During an EMDR session, the patient focuses on a traumatic experience while simultaneously focusing on an external bilateral stimulus [29]. EMDR treatment follows standard EMDR protocols for processing memories of traumatic or stressful life events [30].

\section{Measures}

\section{Diagnoses of SUD and PTSD}

To assess DSM-5 diagnoses of SUD and PTSD, all patients receive a semi-structured face-to-face interview at baseline (T0). The diagnosis of SUD is confirmed using the SUD section of the International Diagnostic Checklists for DSM-IV (IDCL) [31], adapted according to the changed SUD criteria in DSM-V. The IDCL is an established method for the assessment of psychiatric diagnoses. Studies have indicated good clinical practicability and satisfactory to excellent inter-rater and test-retest reliability [31, 32].

The diagnosis of PTSD is confirmed using the Clinician-Administered PTSD Scale (CAPS) [33, 34] for DSM-V [33, 34], which is an updated version of the CAPS for DSM-IV. The CAPS is considered the gold standard for PTSD assessment $[35,36]$. The psychometric properties of the CAPS-IV have been reviewed [35] and indicate excellent convergent and discriminant validity, diagnostic utility, and sensitivity to clinical change.

\section{Primary outcome}

The primary outcome, change from baseline in PTSD symptom severity, is assessed using the CAPS for DSM$\mathrm{V}[33,34]$. The interviewer evaluates the severity of each of the 20 DSM-V PTSD symptoms, based on a combined evaluation of the frequency and intensity on Likert-type scales $(0=$ asymptomatic, $1=\mathrm{mild} /$ subthreshold, $2=$ moderate PTSD/above threshold, $3=$ severe/ markedly increased, 4 =extreme). The 20 DSM-5 PTSD symptom severity scores are then summed up to derive a total severity score, ranging from 0 to 80 . A change of 15 points on the CAPS is considered clinically significant [35].

\section{Seconday outcomes}

Secondary outcomes include change from baseline in severity of substance use, addiction-related problems, dissociative symptoms, depressive symptoms, emotion dysregulation and quality of life (Table 1).

Change from baseline in severity of substance use is measured by the Alcohol Use Disorder Identification Test (AUDIT) $[37,38]$ or the Drug Use Disorder Identification Test (DUDIT) [39, 40], respectively, depending on the type of the patients' primary substance dependence. As additional substance-related outcomes, the mean amount of substance use per day and the number of non-consuming days within the last month is measured with the Timeline Follow Back (TLFB) [41]. Changes in addiction-related problems are assessed by the Addiction Severity Index-Lite (ASI-Lite) [42].

Change in dissociative symptoms (Dissociative Experiences Scale, DES) [25], depressive symptoms (Beck Depression Inventory-II, BDI-II) [43], emotion dysregulation (Difficulties in Emotion Regulation Scale, DERS) [44] and quality of life (Short-Form 12-Item Health Survey, SF-12) [45] are also assessed. The type and severity of early traumatic experiences are measured using the Childhood Trauma Questionnaire (CTQ) [46]. Sociodemographic data of the patients are measured by an interview using a sheet designed according to the documentation standards for the evaluation of addiction treatment [47].

Serious Adverse Events (SAEs; hospitalization, lifethreatening event, disability or permanent damage, death) are measured by SAE Reporting Forms (date; initials of reporting person; participant study number and participant date of birth; SAE description; start date and duration of event; action required; action with regard to intervention; date of last intervention session; causality to intervention or assessment).

\section{Study procedure}

At the patients' admission to the rehabilitation center, a clinical psychologist informs the patient about the study and screens the patient according to the inclusion and exclusion study criteria. Patients that are potentially eligible for the study and consent to participate will receive the baseline assessment (T0) within the first days after admission (Fig. 1). If all inclusion and no exclusion criteria are fulfilled, the patient will be randomized to one of the two study arms. Additional data assessments are conducted at end of treatment (T1), as well as at 3-months and 6-months follow-up (T2 and T3, respectively). The interviews at $\mathrm{T} 0$ and $\mathrm{T} 1$ are conducted faceto-face, the interviews at $\mathrm{T} 2$ and $\mathrm{T} 3$ are conducted by telephone. To promote participant retention and complete follow-up, participants will be contacted several times between the assessments. The coordinating study team monitors the study procedure and traces back potential Serious Adverse Events during the study.

\section{Sample size calculation}

Based on the results of the power analysis of a first RCT examining the effects of a trauma-focused PTSD treatment plus usual substance use treatment vs. usual substance use treatment alone in patients with PTSD and SUD [11], using the Clinician-Administered PTSD Scale (CAPS) as primary outcome, a sample size of 88 participants is required to achieve $80 \%$ power to detect a clinical significant difference of 15 points $(\mathrm{SD}=25)$ in PTSD symptoms at 6 -months 
Table 1 Measures and assessment points used in the study

\begin{tabular}{|c|c|c|c|c|c|c|}
\hline Variable & Measure & Assessment method & T0 Baseline & T1 Post treatment & T2 3 months & T3 6 months \\
\hline SUD diagnosis & IDCL [31] & interview & $x$ & $x$ & $x$ & $x$ \\
\hline PTSD diagnosis & CAPS [33] & interview & $x$ & $x$ & $x$ & $x$ \\
\hline PTSD symptoms & CAPS [33] & interview & $x$ & $x$ & $x$ & $x$ \\
\hline Early traumatic experiences & CTQ [46] & self-report & $x$ & $x$ & $x$ & $x$ \\
\hline Substance use & AUDIT [37]/DUDIT [40] TLFB [41] & interview & $x$ & $x$ & $x$ & $x$ \\
\hline Addiction-related problems & ASI-Lite [42] & interview & $x$ & $x$ & $x$ & $x$ \\
\hline Dissociative symptoms & DES [25] & self-report & $x$ & $x$ & $x$ & $x$ \\
\hline Depressive symptoms & BDI-II [43] & self-report & $x$ & $x$ & $x$ & $x$ \\
\hline Emotion dysregulation & DERS [44] & self-report & $x$ & $x$ & $x$ & $x$ \\
\hline Quality of life & SF-12 [45] & self-report & $x$ & $x$ & $x$ & $x$ \\
\hline Safety & SAE Report Form & self-report & -二—— & -二————— & $x$ & $x$ \\
\hline
\end{tabular}

Notes. IDCL International Diagnostic Checklists. CAPS Clinician-Administered PTSD Scale. CTQ Childhood Trauma Questionnaire. AUDIT Alcohol Use Disorders Identification Test. DUDIT Drug Use Disorders Identification Test. TLFB Timeline Follow Back. ASI-Lite Addiction Severity Index-Lite. DES Dissociative Experience Scale. BDI-II Beck Depression Inventory-II. DERS Difficulties in Emotion Regulation Scale. SF-12 Short-Form 12-Item Health Survey. SAE Serious Adverse Event

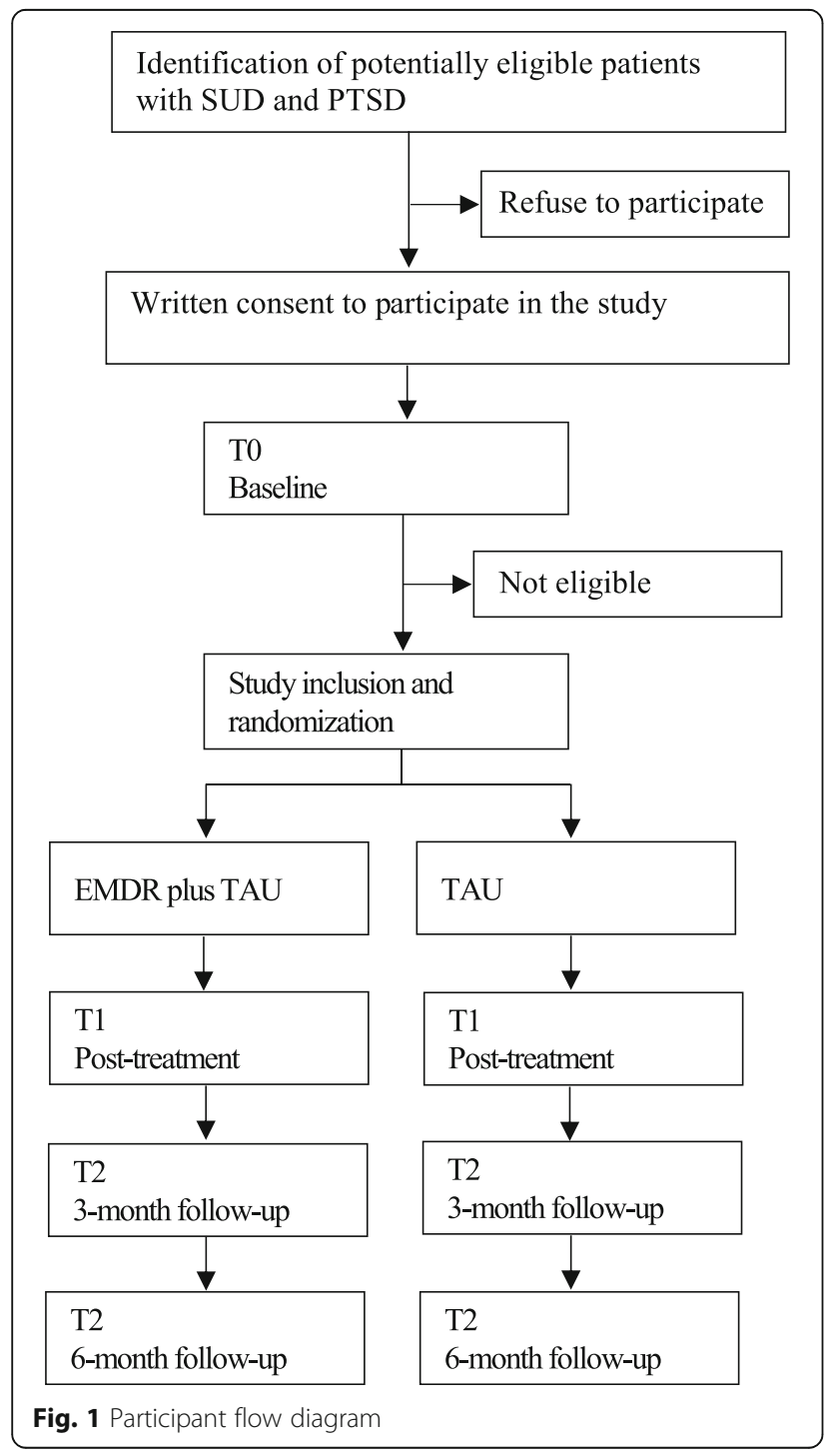

follow-up, using mixed models with a $.05 \alpha$-level of significance. In line with the dropout rates reported in previous intervention studies using rehabilitation inpatients $[48,49]$, a dropout rate of $40 \%$ is expected at 6-month follow-up. Consequently, we aim to include 158 participants in our study.

\section{Statistical analysis}

Summary tables will be provided for all baseline, end of treatment and follow-up variables. Data will be summarized using frequency tables and descriptive statistics (mean, standard deviation, number and percentages of cases). Intention-to-treat and per-protocol analyses will be conducted for all outcomes. Missing data will be imputed using multiple imputation or a similar strategy.

To test the hypothesis that EMDR plus TAU will more greatly reduce PTSD symptoms than TAU alone, a linear mixed model will be calculated. Changes in PTSD symptom severity at 6-month follow-up as measured by the CAPS will be the primary outcome of the study and will be compared between the EMDR plus TAU and TAU group. The analysis will be adjusted for covariates that might be unbalanced between the groups (e.g., sex, type of trauma, treatment time spent in rehabilitation center), as well as for different baseline CAPS scores. The same procedure will be applied for secondary outcomes.

\section{Methods to protect against sources of bias Randomization and allocation}

All patients eligible for the study and providing written consent are randomized to an EMDR or a TAU group in a ratio of 1:1. The generation of the randomization sequence took place prior to the recruitment of participants and was conducted by a researcher of the coordinating site uninvolved in the data assessments of the study (AL). The list for the random assignment of patients to the two treatment arms was generated by the 
randomization software DatInf RandList Version 1.2 using permuted blocks of random sizes between 4 and 10. Each random number is separately stored in sequentially numbered, opaque and sealed envelopes. After the eligibility of a patient for the study has been ensured, the patient is included in the study and the next available randomization number is assigned to the patient in an ascending order.

\section{Blinding}

Study personnel involved in the data assessment of the study are blinded to the patients' treatment assignment. The clinical psychotherapists that deliver the study treatment are not blinded and are therefore uninvolved in the patients' data assessments related to this study. Data analysts are blinded to group allocation.

\section{Treatment fidelity}

All psychotherapists involved in this study regularly work as psychotherapists in the rehabilitation center at which the study is conducted. They were trained in SUD rehabilitation and non-trauma-focused PTSD treatment and completed the EMDR Basic Training program (see http:// www.emdr-europe.org/info.asp?CategoryID=83). All treatments are conducted in line with the respective treatment protocols (see description of interventions). All therapists are supervised by certified EMDR consultants or trainers of EMDR Europe (LCF, PL, AH) during the treatment phase of the study.

Psychotherapists document the content of each individual treatment session in a treatment log, which is monitored by the coordinating site. Out of all individual treatment sessions, $15 \%$ randomly chosen videotaped sessions of each of the two treatment groups are rated for treatment fidelity (compliance with the treatment manual). EMDR treatment adherence is evaluated and monitored by certified EMDR consultants or trainers (LCF, PL, AH) using the EMDR Implementation Treatment Fidelity Scale [50]. Therapist adherence for individual SUD rehabilitation and non-trauma-focused PTSD treatment is evaluated and monitored over the course of study by trained raters of the coordinating site.

\section{Data assessment fidelity}

The personnel involved in data collection (psychology students with at least BSc level) received a 1-day training by the coordinating site prior to the start of the study. The coordinating site monitors the conduction of the data assessments and supervises (AL) the personnel involved in data collection throughout the study. $15 \%$ of the CAPS interviews are reassessed by a second interviewer to establish inter-rater reliability of the PTSD diagnosis and symptom severity. If reliability is low, the interviewers receive additional training. To improve data entry accuracy, all data sheets are entered by using a data scanner. Data entries are checked by data management personnel for correctness.

\section{Ethical and safety issues}

The Ethics Committee of the Medical Council of Hamburg (PV4853) and the Ethics Committee of the Medical Council of Nordrhein (2015233) approved the trial prior to the start of the study. Before patients are included in the study, they are informed about the aims and the design of the study including randomization and the possibility of ending their participation at any time without disadvantages. Potential study participants must provide written informed consent before they can be included in the study. Personal information about potential and enrolled participants will be collected and stored separately from other study data and will be only accessible for the assessors that contact the participants for data assessment. The study dataset does not include personal information and will be analyzed by the coordinating study site. Throughout the study, the standard safety procedures of the inpatient rehabilitation center at which the patients of this study are treated are followed. SAEs are continuously documented throughout the study. SAEs are reported to the coordinating site within $24 \mathrm{~h}$. In case of an unexpected SAE (e.g., life-threatening event, permanent damage or death) over the course of the study, the coordinating site will alert the principal investigator (IS) who will report the SAE to the local ethics committee. The ethics committee and the study team will then decide in accordance with the best interest of the patient if the study procedures are continued or terminated.

\section{Discussion}

This is the first RCT that examines the effectiveness of EMDR combined with TAU (SUD rehabilitation and non-trauma-focused PTSD treatment), in reducing PTSD symptoms in patients with SUD and comorbid PTSD, compared with TAU alone. To test the effectiveness of EMDR within this patient group is crucial, because patients with SUD and comorbid PTSD have been excluded from most clinical trials of trauma-focused interventions for patients with PTSD so far [51]. Consequently, there is limited evidence that EDMR is effective in this patient group. As a secondary outcome, we also examine the effectiveness of EMDR on substance use. As patients may use substances to regulate PTSDrelated symptoms $[12,52]$, it could be expected that trauma-focused treatment might also reduce SUD symptoms.

\section{Strengths and limitations}

A strength of this study is that we will be able to include a sufficient sample size in our study to detect group 
differences between the EMDR plus TAU and the TAU group. However, we expect a high drop-out rate in our sample of patients with comorbid mental disorders. The expected high drop-out rate might complicate the interpretation of the study results. A strength of this study might be that the patients included in this study live in various parts of Germany, represent a great variety of their sociodemographic backgrounds and were exposed to different types of traumatic experiences. This may increase the generalizability of our results. On the other hand, we exclude patients that are younger than 18 or older than 65 years; don't speak German; present acute suicidal, psychotic or severe dissociative symptoms; or show severely cognitive impairment. The study findings might not be generalized to these populations of patients with SUD and PTSD.

In summary, the primary aim of this study is to investigate the effectiveness of EMDR, added to usual SUD rehabilitation and non-trauma-focused PTSD treatment (TAU), in reducing PTSD symptoms, compared with TAU alone. If this RCT proves that EMDR is effective in patients with SUD and comorbid PTSD, the results of this RCT may encourage a more frequent use of evidence-based trauma-focused approaches in routine treatment of patients with SUD and comorbid PTSD.

\section{Trial status}

We are recruiting patients and started with data collection. The first patient was enrolled in September 2015.

\section{Abbreviations}

AE: Adverse events; ASI-Lite: Addiction Severity Index-Lite; AUDIT: Alcohol Use Disorders Identification Test; BDI-II: Beck Depression Inventory-II; CAPS: Clinician-Administered PTSD Scale; DERS: Difficulties in Emotion Regulation Scale; DES: Dissociative Experience Scale; DSM-V: Diagnostic and Statistical Manual of Mental Disorders; DUDIT: Drug Use Disorders Identification Test; EMDR: Eye Movement Desensitization and Reprocessing; IDCL: International Diagnostic Checklists; PTSD: Posttraumatic stress disorder; RCT: Randomized controlled trial; SAE: Serious Adverse Event; SD: Standard deviation; SF-12: Short-Form 12-Item Health Survey; TAU: Treatment as usual; TLFB: Timeline Follow Back

\section{Acknowledgements}

We greatly thank Jehanra Moriabadi, Helen Seeliger, Bella Liebig and Alexandra Smyth for preparing the study documents; Eike Neumann-Runde for setting up an online monitoring system, Lea Tichelbäcker for screening patients; Nina Lejeune, Alexandra Smyth, Marianne Haar and Julia Kraus for their tremendous efforts in the data collection; Isabell Liebig and Hannah Beckmann for supporting the study monitoring; Dr. Philipp Hiller for supervising the scanning of the data sheets and the plausibility checks; and Henriette Reichwald for conducting the data plausibility checks. We greatly thank all psychotherapists involved at any stage of the study that provide EMDR and/or usual treatment: Annika Deckers, Karin Heim, Silvia Hollweger, Elisabeth Mick, Christina Rohde, Marieke Schüberl and Lea Tichelbäcker.

\section{Funding}

This study is supported by a grant from the Society of Rehabilitation Sciences North Rhine Westphalia, Germany (grant no. GfR13007/623-18, E-Mail: info@rehaforschung-nrw.de) and is co-funded by a grant from the European EMDR Society (E-Mail: info@emdr-europe.org). The study sponsors did not influence the study design, data collection, management, analysis, and interpretation of data; writing of the study protocol; or the decision to submit the study protocol for publication.

\section{Availability of data and materials \\ Not applicable.}

\section{Authors' contributions}

IS is the PI of the trial and was primarily responsible for the design of the study. AL, LCF, AH and PL contributed to the study design. LCF supervises the study therapists. AL coordinates the trial and drafted the manuscript. IS, $\mathrm{LCF}, \mathrm{AH}, \mathrm{PL}$ and $\mathrm{GM}$ contributed to editing the manuscript and read and approved the final manuscript.

\section{Competing interests}

The study received a grant of $10.000 €$ by EMDRIA Europe. AH heads the EMDR-Institute Germany that trains in EMDR. He is a board member of the EMDR-Institute Germany. LCF is a board member of the EMDR-Institute Germany and an EMDR consultant. PL is a member of the EMDR-Institute Germany. He is treasurer of EMDR Europe. The remaining authors have no competing interests.

\section{Consent for publication}

Not applicable.

\section{Ethics approval and consent to participate}

The Ethics Committee of the Medical Council of Hamburg (PV4853) and the Ethics Committee of the Medical Council of Nordrhein (2015233) approved the trial prior to the start of the study. Potential study participants must provide written informed consent before they can be included in the study. Before patients are included in the study, they are informed about the aims and the design of the study including randomization and the possibility of ending their participation at any time without disadvantages.

\section{Publisher's Note}

Springer Nature remains neutral with regard to jurisdictional claims in published maps and institutional affiliations.

\section{Author details}

${ }^{1}$ Department of Psychiatry and Psychotherapy, University Medical Center Hamburg-Eppendorf, Hamburg, Germany. ${ }^{2}$ Center for Interdisciplinary Addiction Research, Department of Psychiatry and Psychotherapy, University Medical Center Hamburg-Eppendorf, University of Hamburg, Martinistr. 52, Hamburg D-20246, Germany. ${ }^{3}$ AHG Clinic Dormagen, Dormagen, Germany. ${ }^{4}$ EMDR-Institute Germany, Bergisch Gladbach, Germany.

Received: 8 February 2017 Accepted: 4 March 2017

Published online: 16 March 2017

\section{References}

1. Dore G, Mills K, Murray R, Teesson M, Farrugia P. Post-traumatic stress disorder, depression and suicidality in inpatients with substance use disorders. Drug Alcohol Rev. 2012;31:294-302.

2. Driessen M, Schulte $S$, Luedecke C, Schaefer I, Sutmann F, Ohlmeier M, et al. Trauma and PTSD in patients with alcohol, drug, or dual dependence: a multi-center study. Alcohol Clin Exp Res. 2008:32:481-8.

3. Mills KL, Teesson M, Ross J, Peters L. Trauma, PTSD, and substance use disorders: findings from the Australian National Survey of Mental Health and Well-Being. Am J Psychiatry. 2006;163:652-8.

4. Potthast N, Catani C. Trauma und Sucht: Implikationen für die Psychotherapie [trauma and addiction: implications for psychotherapy]. SUCHT. 2012;58:227-35.

5. Schäfer I, Schulze C, Stubenvoll M. Psychotherapie bei Abhängigkeitserkrankungen und Posttraumatischer Belastungsstörung [psychotherapy for substance use disorders and posttraumatic stress disorder]. SUCHT. 2011;57:353-61.

6. Flatten G, Gast U, Hofmann A, Knaevelsrud C, Lampe A, Liebermann P, et al. Posttraumatische Belastungsstörung: S3-Leitlinie und Quellentexte. Stuttgart: Schattauer; 2013.

7. National Collaborating Centre for Mental Health. Post-traumatic stress disorder: the management of PTSD in adults and children in primary and secondary care. London: Gaskell and the British Psychological Society; 2005. 
8. Australian Center for Posttraumatic Health (ACPMH). Australian guidelines for the treatment of acute stress disorder \& posttraumatic stress disorder. Guidelines Appendices. Melbourne, VIC: ACPMH; 2013. Retrieved from http://phoenixaustralia.org/resources/ptsd-guidelines/.

9. Bradley R, Greene J, Russ E, Dutra L, Westen D. A multidimensional metaanalysis of psychotherapy for PTSD. Am J Psychiatry. 2005;162:214-27.

10. Watts BV, Schnurr PP, Mayo L, Young-Xu Y, Weeks WB, Friedman MJ. Metaanalysis of the efficacy of treatments for posttraumatic stress disorder. J Clin Psychiatry. 2013;74:e541-50.

11. Mills KL, Teesson M, Back SE, Brady KT, Baker AL, Hopwood S, et al. Integrated exposure-based therapy for co-occurring posttraumatic stress disorder and substance dependence: a randomized controlled trial. JAMA J Am Med Assoc. 2012;308:690-9.

12. Coffey SF, Stasiewicz PR, Hughes PM, Brimo ML. Trauma-focused imaginal exposure for individuals with comorbid posttraumatic stress disorder and alcohol dependence: revealing mechanisms of alcohol craving in a cue reactivity paradigm. Psychol Addict Behav. 2006;20:425-35.

13. van Dam D, Ehring T, Vedel E, Emmelkamp PM. Trauma-focused treatment for posttraumatic stress disorder combined with CBT for severe substance use disorder: a randomized controlled trial. BMC Psychiatry. 2013;13:172.

14. Foa EB, Yusko DA, McLean CP, Suvak MK, Bux DA, Oslin D, et al. Concurrent naltrexone and prolonged exposure therapy for patients with comorbid alcohol dependence and PTSD: a randomized clinical trial. JAMA. 2013;310:488-95.

15. McGovern MP, Lambert-Harris C, Xie H, Meier A, McLeman B, Saunders E. A randomized controlled trial of treatments for co-occurring substance use disorders and post-traumatic stress disorder. Addiction. 2015;110:1194-204.

16. Becker CB, Zayfert C, Anderson E. A survey of psychologists' attitudes towards and utilization of exposure therapy for PTSD. Behav Res Ther. 2004:42:277-92

17. Najavits LM. Treatment for the dual diagnosis of posttraumatic stress and substance use disorders. Dir Addict Treat Prev. 2004:8:1-11.

18. Regel S, Joseph S. Review of post-traumatic stress disorder: the management of PTSD in adults and children in primary and secondary care. Couns Psychother Res. 2007;7:129-30.

19. Back SE, Brady KT, Sonne SC, Verduin ML. Symptom improvement in cooccurring PTSD and alcohol dependence. J Nerv Ment Dis. 2006;194:690-6.

20. Ouimette $P$, Read JP, Wade M, Tirone V. Modeling associations between posttraumatic stress symptoms and substance use. Addict Behav. 2010;35:64-7.

21. Saladin ME, Drobes DJ, Coffey SF, Dansky BS, Brady KT, Kilpatrick DG. PTSD symptom severity as a predictor of cue-elicited drug craving in victims of violent crime. Addict Behav. 2003;28:1611-29.

22. Hien DA, Jiang H, Campbell AN, Hu M-C, Miele GM, Cohen LR, et al. Do treatment improvements in PTSD severity affect substance use outcomes? A secondary analysis from a randomized clinical trial in NIDA's Clinical Trials Network. Am J Psychiatry. 2009;167:95-101.

23. Waldrop AE, Back SE, Verduin ML, Brady KT. Triggers for cocaine and alcohol use in the presence and absence of posttraumatic stress disorder. Addict Behav. 2007;32:634-9.

24. American Psychiatric Association. Diagnostic and Statistical Manual of Mental Disorders: DSM-5. Washington, D.C.: American Psychiatric Association; 2013

25. Spitzer C, Freyberger HJ, Stieglitz R-D, Carlson EB, Kuhn G, Magdeburg N, et al. Adaptation and psychometric properties of the German version of the dissociative experience scale. J Trauma Stress. 1998;11:799-809.

26. Reddemann L. Imagination als heilsame Kraft zur Behandlung von Traumafolgen mit ressourcenorientierten Verfahren. 8th ed. Stuttgart: KlettCotta; 2003.

27. Wagner AW, Linehan MM. Applications of dialectical behavior therapy to posttraumatic stress disorder and related problems. Cogn Behav Ther Trauma. 2006;2:117-45

28. Najavits LM, Schäfer I, Stubenvoll M, Dilling A. Posttraumatische Belastungsstörung und Substanzmissbrauch: Das Therapieprogramm "Sicherheit finden". 1st ed. Göttingen: Hogrefe; 2008.

29. Shapiro F. Eye movement desensitization and reprocessing procedure: from EMD to EMDR: a new treatment model for anxiety and related traumata. Behav Ther. 1991;14:133-5.

30. Shapiro F, Forrest MS. Eye movement desensitization and reprocessing (EMDR): Basic principles, protocols and procedures. New York: Guilford Press; 2001.
31. Hiller W, Zaudig M, Mombour W. Internationale Diagnosen Checklisten (IDCL) für DSM-IV. Göttingen: Hogrefe; 1997.

32. Hiller W, Zaudig M, Mombour W. Development of diagnostic checklists for use in routine clinical care: a guideline designed to assess DSM-III-R diagnoses. Arch Gen Psychiatry. 1990;47:782-4.

33. Schnyder U. Clinician-Administered PTSD Scale for DSM-5 - Past month version. Zürich: UniversitätsSpital Zürich; 2013.

34. Weathers FW, Blake DD, Schnurr PP, Kaloupek DG, Marx BP, Keane TM. The Clinician-Administered PTSD Scale for DSM-5 (CAPS-5). National Center for PTSD; 2013. Retrieved from http://www.ptsd.va.gov/professional/ assessment/adult-int/caps.asp.

35. Weathers FW, Keane TM, Davidson JR. Clinician-administered PTSD scale: a review of the first ten years of research. Depress Anxiety. 2001;13:132-56.

36. Weiss DS. Structured clinical interview techniques for PTSD. In: Wilson JP, Keane TM, editors. Assessing psychological trauma and PTSD. 2nd ed. New York: Guilford Press; 2004. p. 103-21.

37. Dybek I, Bischof G, Grothues J, Reinhardt S, Meyer C, Hapke U, et al. The reliability and validity of the alcohol use disorders identification test (AUDIT) in a German general practice population sample. J Stud Alcohol. 2006;67:473-81.

38. Saunders JB, Aasland OG, Babor TF, De la Fuente JR, Grant M. Development of the alcohol use disorders identification test (AUDIT): WHO collaborative project on early detection of persons with harmful alcohol consumption-II. Addiction. 1993;88:791-804.

39. Berman AH, Bergman H, Palmstierna T, Schlyter F. Evaluation of the Drug Use Disorders Identification Test (DUDIT) in criminal justice and detoxification settings and in a Swedish population sample. Eur Addict Res. 2005:11:22-31.

40. Thormann A, Schalast N. Drug Use Disorders Identification Test (DUDIT). Duisburg: Institute of Forensic Psychiatry, University of Duisburg-Essen; 2002.

41. Sobell LC, Sobell MB. Timeline follow-back: A technique for assessing selfreported alcohol consumption. In: Litten RZ, Allen JP, editors. Measuring alcohol consumption: Psychosocial and biochemical methods. Totowa: Humana Press; 1992. p. 41-72.

42. McGahan PL, Griffith JA, Parente R, McLellan AT. Addiction Severity Index composite scores manual. Philadelphia: Treatment Research Institute; 1986.

43. Hautzinger $\mathrm{M}$, Keller F, Kühner C. Beck Depressions-Inventar (BDI-II). Frankfurt am Main. Germany: Harcourt Test Services; 2006.

44. Gratz KL, Roemer L. Multidimensional assessment of emotion regulation and dysregulation: Development, factor structure, and initial validation of the difficulties in emotion regulation scale. J Psychopathol Behav Assess. 2004:26:41-54.

45. Ware Jr JE, Kosinski M, Keller SD. A 12-item short-form health survey: construction of scales and preliminary tests of reliability and validity. Med Care. 1996:34:220-33.

46. Bernstein DP, Stein JA, Newcomb MD, Walker E, Pogge D, Ahluvalia T, et al. Development and validation of a brief screening version of the childhood trauma questionnaire. Child Abuse Negl. 2003:27:169-90.

47. Deutsche Gesellschaft für Suchtforschung und Suchttherapie [German Society for Addiction Research and Therapy]. Dokumentationsstandards III für die Evaluation der Behandlung von Abhängigen [Documentation standards III for the evaluation of addiction treatment]. Sucht. 2001:47:3-94.

48. Missel P, Schneider B, Bachmeier R, Funke W, Garbe D, Herder F, et al. Effektivität der stationären Suchtrehabilitation - FVS-Katamnese des Entlassjahrgangs 2006 von Fachkliniken für Alkohol-und Medikamentenabhängige. Sucht Aktuell. 2010;17:9-20.

49. Zobel M, Missel P, Bachmeier R, Funke W, Garbe D, Herder F, et al. Effektivität der stationären Suchtrehabilitation-FVS-Katamnese des Entlassjahrgangs 2004 von Fachkliniken für Alkohol-und Medikamentenabhängige. Sucht Aktuell. 2005;12:5-15.

50. Korn DL, Zangwill W, Lipke H, Smyth NJ. EMDR Implementation Fidelity Scale. Austin: EMDRIA; 2001.

51. van Minnen A, Harned MS, Zoellner L, Mills K. Examining potential contraindications for prolonged exposure therapy for PTSD. Eur J Psychotraumatology. 2012;3:18805.

52. Jacobsen LK, Southwick SM, Kosten TR. Substance use disorders in patients with posttraumatic stress disorder: a review of the literature. Am J Psychiatry. 2001;158:1184-90. 